Carmine IORIO* - Paolo FONTANA** - Marco BARDIANI*** - Roberto SCHERINI**** - Bruno MASSA*****

\title{
Nuovi dati sulla distribuzione in Italia di alcuni Ortotteri (Orthoptera, Ensifera, Caelifera)
}

\begin{abstract}
Riassunto: Vengono riportati nuovi dati sulla distribuzione in Italia di 10 specie di Ortotteri (5 Tettigoniidae, 2 Gryllidae e 3 Acrididae), non segnalate in precedenza per alcune regioni o poco note.
\end{abstract}

Abstract: Updating of the Italian distribution of some Orthoptera (Ensifera, Caelifera).

New records are reported on the distribution in Italy of 10 Orthoptera (5 Tettigoniidae, 2 Gryllidae and 3 Acrididae), previously unreported or less known from some Italian regions.

Key words: Orthoptera, Ensifera, Caelifera, distribution, Italy.

\section{INTRODUZIONE}

Nel presente contributo si rendono noti nuovi dati faunistici relativi ad alcune specie di Ortotteri della fauna italiana, con lo scopo di arricchire le conoscenze sulla loro distribuzione geografica in Italia, per alcune di esse tuttora piuttosto frammentarie. Tali reperti rappresentano le prime segnalazioni a livello regionale o riguardano specie la cui distribuzione è poco nota. I dati presentati si riferiscono a materiale raccolto sul campo o all'esame di collezioni museali. Per ogni specie vengono riportate le indicazioni sulle stazioni di rinvenimento.

Collezioni in cui è conservato il materiale citato $\mathrm{BMCP}=$ Collezione Bruno Massa, Palermo $\mathrm{CCCP}=$ Collezione Claudio Codato, Nervesa della Battaglia (TV).

$\mathrm{CICP}=$ Collezione Carmine Iorio, Bologna $\mathrm{MBCP}=$ Collezione Marco Bardiani, Marcaria $(\mathrm{MN})$ $\mathrm{MSNG}=$ Museo Civico di Storia Naturale 'G. Doria', Genova

RMNH=Naturalis (National Museum of Natural History), Leiden (NL)

\section{ELENCO DELLE SPECIE}

\section{Tettigoniidae}

Leptophyes laticauda (Frivaldsky, 1867)

REPERTI: Lazio, Mentana (Roma), Riserva Naturale della Macchia di Gattaceca, 2.VII.2011, 1, C. Piredda leg. (CICP). Nuova per il Lazio.

Montana stricta (Zeller, 1849)

RePERTI: Veneto, Crocetta del Montello (Treviso), Grave di Ciano, 17.VIII.2015, 1\%, Luisa De Savi leg. (CCCP). Prima conferma per il Veneto, di specie già citata per Parona di Valpolicella (VR), per un reperto raccolto nel 1938 (MSNG) (Baroni et al., 2016).

Bicolorana bicolor bicolor (Philippi, 1830)

RePERTI: Basilicata, Terranova di Pollino (Potenza), sopra Lago Fondo, pendici di Serra Di Crispo (1600 m), 6.VII.2012, 1 ㅇ olottera, B. Massa (BMCP). Nuova per la Basilicata, sono noti pochi reperti per gli Appennini, i più meridionali in Abruzzo (Massa et al., 2012).

\footnotetext{
${ }^{*}$ Carmine Iorio, Via Scandellara 58, 40138 Bologna, Italia. E-mail: ioriocarmine01@gmail.com

** Paolo Fontana, Fondazione Edmund Mach - Centro Trasferimento Tecnologico, Unità operativa: Protezione delle piante agroforestali e apicoltura, Via della Val, 2 - Loc. Costa di Casalino, 38057 Pergine Valsugana (TN), Italia. E-mail: paolo_api.fontana@fmach.it

*** Marco Bardiani, MiPAAF, Corpo Forestale dello Stato, Centro Nazionale per lo Studio e la Conservazione della Biodiversità Forestale "Bosco Fontana" di Verona, Sede di Bosco Fontana, Strada Mantova, 2946045 Marmirolo (MN), Italia. E-mail: bardianimarco@gmail.com

${ }^{* * * * *}$ Roberto Scherini, Via Pianzola 14, 27010 Linarolo (PV), Italia. E-mail: rscherini@gmail.com

${ }^{* * * * *}$ Bruno Massa, Dipartimento di Scienze agrarie, alimentari e forestali, Viale Scienze Ed. 5A, 90128 Palermo, Italia. E-mail: bruno.massa@unipa.it
} 
Sepiana sepium (Yersin, 1854)

REPERTI: Lombardia, Linarolo (Pavia), 15.VIII.2017, $\hat{\jmath} \mathrm{e}$ +, R. Scherini leg. (BMCP). Prima segnalazione per la Lombardia.

Incertana drepanensis (Massa, Fontana, Buzzetti, 2006)

REPERTI: Sicilia, Menfi (Agrigento) 26.IX.1971, $q$, M.C. \& G. Kruseman (già Zoologisch Museum Amsterdam, ora RMNH) (Luc Willemse, com. pers.). Di questa specie, unico rappresentante del genere in Italia, erano note due località siciliane, saline di Trapani (locus typicus) e Piana degli Albanesi, loc. Maganoce (Palermo) (Massa et al., 2012).

\section{Gryllidae}

Stenonemobius gracilis (Jakovleff, 1871)

REPERTI: Lazio (Roma), Litorale Tenuta Castelporziano VIII.2014, P. Maltzeff (Malaise trap); Marcaria (Mantova) loc. S. Michele in bosco, $25 \mathrm{~m}$, 30.VIII.2013 trovati resti dentro ragnatela, all'interno di abitazione, M. Bardiani leg. 2.VIII.2015 e 10.VIII.2015 all'interno di abitazione, alla sera (MBCP). Il reperto di Castelporziano costituisce la prima segnalazione per il Lazio, mentre i ritrovamenti di Marcaria risultano nuovi per la Lombardia.
Gryllomorpha (Gryllomorphella) uclensis (Pantel, 1890)

REPERTI: Puglia, Alberobello (Bari), 18.VIII.2017, 10ิ, C. Iorio leg. (CICP). Prima segnalazione per la Puglia.

\section{Acrididae}

Odontopodisma decipiens insubrica Nadig, 1980 REPERTI: Emilia-Romagna, Pianoro Vecchio (Bologna), 27.VI.2013, diversi 해소 e 우우, C. Iorio leg. (CICP). Nuova per l'Emilia-Romagna.

Heteracris annulosa (Walker, 1870)

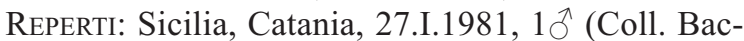
cetti, MSNG). Nuova per la Sicilia, ma nota per le isole del Canale di Sicilia e per il sud della Sardegna (Cillo et al., 2012; Massa et al., 2012).

Oedipoda germanica germanica (Latreille, 1804) REPERTI: Sicilia, Laghetto di Faro (Messina), 7.VIII.1989, 1ㅇ, B. Massa leg. (BMCP). Nuova per la Sicilia.

\section{RINGRAZIAMENTI}

Ringraziamo sinceramente Claudio Codato, Claudio Piredda e Luc Willemse per le segnalazioni inedite che gentilmente ci hanno comunicato.

\section{BIBLIOGRAFIA}

Baroni D., Chemollo M., Bonifacino M., 2016 - Nuove segnalazioni di Montana stricta (Zeller, 1849) per l'Italia settentrionale (Orthoptera Tettigoniidae). Rivista del Museo Civico di Scienze Naturali “E. Caffi” Bergamo, 29: 45-50.

Cillo D., Bazzato E., Fols F., 2012 - Segnalazioni faunistiche 546. Heteracris annulosa Walker, 1870 (Orthoptera Acrididae). Bollettino della Società entomologica italiana, 144(2): 89-92.

Massa B., Fontana P., Buzzetti F.M., Kleukers R., Odé B., 2012 - Orthoptera. Fauna d'Italia. Vol. XLVIII. Calderini, Bologna, 563 pp. + DVD 